\title{
Carnets
}

Revue électronique d'études françaises de l'APEF

Deuxième série - 9 | 2017

Reconnaissances et légitimité en français

\section{Les débuts du surréalisme français : reconnaissance littéraire et reconnaissance médiatique}

Paul Aron

\section{OpenEdition}

Journals

Édition électronique

URL : http://journals.openedition.org/carnets/2005

DOI : $10.4000 /$ carnets. 2005

ISSN : 1646-7698

Éditeur

APEF

Référence électronique

Paul Aron, «Les débuts du surréalisme français : reconnaissance littéraire et reconnaissance médiatique », Carnets [En ligne], Deuxième série - 9 | 2017, mis en ligne le 31 janvier 2017, consulté le 30 avril 2019. URL : http://journals.openedition.org/carnets/2005; DOI : 10.4000/carnets.2005

Ce document a été généré automatiquement le 30 avril 2019.

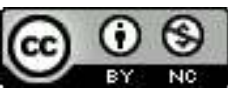

Carnets est mis à disposition selon les termes de la licence Creative Commons - Atribution - Pas d'utilisation commerciale 4.0 International. 


\title{
Les débuts du surréalisme français : reconnaissance littéraire et reconnaissance médiatique
}

\author{
Paul Aron
}

1 Les avant-gardes sont des ruptures autoproclamées dans le continuum des pratiques artistiques et culturelles. Elles se développent toujours à la marge des groupes ou des catégories existantes. Elles en mettent la légitimité en question, par un rejet de leurs codes et comportements, ou en déplaçant les lignes qui fondaient leurs normes. Par définition, les avant-gardes s'imposent dès lors comme des contestations minoritaires avant de connaître parfois à leur tour une reconnaissance ou une consécration durables.

2 Dans le schéma de la production culturelle proposée par Pierre Bourdieu (1992), la problématique des avant-gardes est décrite en faisant principalement appel à deux concepts. (1) Le champ de production restreinte, que Bourdieu désigne aussi comme une production culturelle destinée aux producteurs eux-mêmes, semble le lieu privilégié de l'émergence du phénomène. Le public auquel se destinent les productions avant-gardistes est à la fois un public averti des normes existantes et qui peut accepter de les voir contestées. (2) L'avant-gardisme concerne également les frontières qui séparent les disciplines du monde artistique autant que celles qui séparent l'art du non-art (l'artisanat, par exemple). Les pratiques d'avant-garde mettent souvent l'accent sur le caractère conventionnel de ces frontières et des codes artistiques qu'elles instituent; elles visent également à en modifier les tracés.

3 Ainsi cernées dans l'espace social des productions artistiques, les pratiques d'avant-garde sont décrites comme investissant les vecteurs d'expression propres au champ restreint: petites revues, activités cénaculaires, expositions et manifestes à diffusion limitée mais ciblée. Il serait cependant tout à fait erroné de croire qu'elles aient ignoré ou qu'elles se soient développées à l'écart des médias plus traditionnels ou dominants. Ce phénomène peut déjà être observé au xix ${ }^{\mathrm{e}}$ siècle, notamment dans les publications satiriques ou ironiques qui accompagnent toutes les innovations artistiques. C'est le cas du naturalisme, dont la presse accompagne chacune des manifestations (Grand-Carteret, 
1905), ou bien encore du symbolisme dont les formes de prédilection sont moquées jusque dans les cafés-concerts (Grojnowski, 1997). Mais après la première guerre mondiale, le phénomène prend une nouvelle expansion, à la mesure de la place grandissante que la presse occupe dans l'espace public (Aron, Espagnon, 2009). Celle-ci atteint alors le sommet de sa puissance, puisqu'elle a le monopole de l'information avant le développement de la radio. Nombre de quotidiens ont des pages et des chroniques culturelles spécialisées. Il en est même qui se consacrent exclusivement à la vie artistique.

L'exemple que je voudrais développer à ce propos est celui de l'émergence du surréalisme dans les années 1919-1924, soit donc dans les années où le dadaïsme devient le surréalisme au prix d'une transformation profonde du leadership, des modes de fonctionnement du groupe et des domaines qu'il investit. Cette évolution se donne évidemment à lire dans les petites revues spécialisées du champ restreint, mais également, il faut y insister, dans la grande presse qui diffuse publiquement les termes stratégiques dont usent les avant-gardes, et met en évidence la concurrence des agents qui s'en revendiquent ${ }^{1}$. Ce que le dadaïste belge Clément Pansaers appelait le «tam-tam ", le goût de la réclame, est une composante essentielle de l'histoire de ces groupes. On ne peut donc décrire leur émergence en se focalisant sur le seul secteur restreint de la production culturelle.

5 Récemment mis en ligne et bénéficiant d'une attention soutenue de la part de plusieurs chercheurs $^{2}$, le quotidien artistique Comcedia offre à cet égard un point de vue particulièrement intéressant. Comœdia est, au lendemain de la Grande Guerre, un grand journal quotidien, désireux d'atteindre le public « moyen » des théâtres, du cinéma et des spectacles en tous genres. Selon l'Histoire générale de la presse française, «il restait en marge des grands courants littéraires de la période» (Bellanger, 1972: 595). C'est précisément le contraire que je voudrais montrer.

6 Pendant cette période, le journal est dirigé par George Casella du $1^{\text {er }}$ septembre 1919 à la fin de l'année 1922, date à laquelle il meurt. Il est ensuite publié sous la direction de Gabriel Alphaud, avec Fernand Gregh et Raymond Charpentier comme rédacteurs en chef. En 1929, il est racheté par Jean de Rovera. En matière artistique, ce quotidien défend une vision traditionnelle, voire réactionnaire, en phase avec les goûts du grand public auquel il est destiné : musique légère, danse, opéras et opérettes, expositions officielles y occupent largement l'espace rédactionnel. Il faut y voir non pas un lieu neutre comme semblent l'avoir conçu les éditeurs scientifiques modernes de Breton ou de Picabia, mais bien un agent à part entière du champ culturel, et un agent mû par la double logique de son économie particulière : celle d'une entreprise de presse, qui doit assurer le salaire de ses journalistes, payer ses bureaux, son imprimeur, et celle d'un organe de référence légitimé par le statut de ses éditorialistes et de ses grandes signatures, qui sont le garant de son autorité. Une part de l'histoire de l'avant-garde se construit donc dans un paradoxe médiatique : faire connaitre des manifestations culturelles contestatrices dans un lieu qui résiste par nature à celle-ci.

\section{Une tribune paradoxale}

7 Sans reprendre ici la chronologie de l'émergence du dadaïsme à Paris, si bien retracée par Michel Sanouillet, il faut insister sur le rôle qu'y a joué Picabia. Comme Duchamp, ce peintre vient d'un milieu pratiquant les arts. Remarqué très jeune pour ses toiles postimpressionnistes, ami intime des Pissaro, sa première exposition personnelle en 1905 
remporte un grand succès. Sa fortune personnelle lui permet ensuite de rompre avec ses premiers choix esthétiques et de s'ouvrir aux recherches plus contemporaines. L'héritage de sa mère lui donne champ libre. En 1911, il collabore avec Jacques Villon et connaît le succès à l'exposition de l'Armory Show de New York en 1913 en même temps que Duchamp. Il y fonde la revue 291 avec ce dernier et Man Ray, puis 391 à Barcelone en 1916. En 1918, il rencontre Tzara à Zurich, soutient le dadaïsme, puis rompt publiquement avec lui en 1921. Il illustre ensuite les premières livraisons de la revue Littérature, avant de reprendre une vie marquée par de nombreuses conquêtes féminines, le jeu, le cinéma, la photographie et le monde du spectacle (notamment les ballets suédois pour lesquels il réalise des décors).

8 La dimension mondaine de ce parcours fait comprendre les liens qui pouvaient exister entre Picabia et le milieu de la grande presse culturelle (Sanouillet, 1979 : 407). Georges Casella figure parmi les signataires du célèbre tableau L'Eil cacodylate. C'est de toute évidence grâce à lui que le journal se montre particulièrement attentif à toutes les manifestations qui impliquent le célèbre peintre. Dès février 1920, le journaliste Jean Valmy-Baysse fait écho à la lecture des sept manifestes Dada au Salon des Indépendants ${ }^{3}$. À la fin du mois, il évoque l'exclusion des dadaïstes par les cubistes qui n'exposeront pas à la section d'or le 2 mars. Bien informé, le journaliste précise : « Mais nous ne perdrons pas pour attendre: les exclus feront prochainement une grande exposition avec manifestations littéraires et spectacles dadas. ${ }^{4}$ " Selon Casella, les dadas - sauf Tzara et Picabia - sont des farceurs, mais ils ont bien le droit de s'amuser ${ }^{5}$. Cette opinion bienveillante se heurte toutefois à la véhémence de quelques lecteurs. Le cas le plus notable est celui de Rachilde qui se lance dès le $1^{\mathrm{er}}$ avril 1920 dans une dénonciation en règle du mouvement à qui elle reproche d'être animé par des étrangers et, en particulier, par les Allemands. L'argument, qui sera souvent repris, est évidemment à double portée, puisqu'il consiste à refuser une dynamique artistique au nom des valeurs patriotiques répandues dans l'opinion publique. Picabia et Breton bénéficient pourtant de la même tribune pour répondre à l'animatrice du Mercure de France. Le premier insiste sur son rôle dans le dadaïsme, ne voulant pas se laisser distancer par Tzara. Quant à Breton, il compare le Dadaïsme au Symbolisme qui eut, lui aussi, à subir bien des attaques. La flèche ad feminam insiste sur la jeunesse du groupe : «Il est certain que nous 'courons à la mort' comme tout le monde, mais nous avons la chance d'être les derniers partis. » L'article est signé : « André Breton, directeur de Littérature, revue dadaïste ». (Bonnet, $1988: 629)^{6}$

Cet échange met en place une sorte de jeu de rôles qui sera reconduit à maintes reprises dans le journal. Celui-ci accorde aux manifestations avant-gardistes une place relativement discrète puisqu'aucune rubrique ne leur est consacrée. Le commentaire et les légendes des photographies marquent aussi de la distance, mais l'information est donnée. Un journaliste ou un correspondant réagissent avec véhémence pour réaffirmer une doxa plus traditionnelle, et le journal donne alors la parole aux accusés pour qu'ils se défendent. Sous Casella, Comodia se déclare ouvert aux nouveautés; par après, on le verra, le journal adoptera des positions plus hostiles.

Picabia a bien noté cette évolution en rendant hommage à son ami, en 1922 :

Je félicite vivement la nouvelle direction de Comodia! Quel beau journal, plein de belles conneries! Il est vrai que M. Léon Bérard a écrit une belle lettre et que nous avons pu admirer sa belle figure, mais on cherche vainement dans les six belles pages un petit coin de lumière: rien, c'est le noir le plus absolu, mon pauvre Casella, Comoedia porte ton deuil.

S'ils ont copié l'en-tête et les caractères du Figaro, ils ont moins de pudeur que dans 
ce journal! Ils n'ont même pas songé à y laisser ton nom, au moins pendant quelque temps. Il est vrai que c'est une consolation de ne pas te voir figurer là... (Picabia, $1922: 3)$

11 Comme Casella, les journalistes et collaborateurs du journal spécialisé dans les matières artistiques qu'est Comœdia ne sont évidemment pas de simples témoins des conflits du champ culturel. Même s'ils n'ont pas toujours laissé une trace marquante dans l'histoire, il est possible d'identifier certains d'entre eux. Comme on le verra, la plupart étaient actifs dans le monde musical et dans le théâtre.

12 Jean Valmy-Baysse (1874-1962), déjà cité, a écrit de la poésie, des chansons et des pièces de théâtre avant de devenir l'historien de la Comédie française dont il fut secrétaire général entre 1927 et 1933. On lui doit plusieurs livres, principalement anecdotiques, sur la presse du siècle précédent (notamment sur André Gill et Gustave Doré), sur des peintres (plutôt académiques comme Jean-Paul Laurens) et sur la vie parisienne ( $\mathrm{La}$ curieuse aventure des boulevards extérieurs, 1950). C'est aussi un ancien combattant, sensible, comme nombre de ses contemporains, à l'impossibilité de partager l'expérience des tranchées avec ceux qui ne l'ont pas vécue : il en fera le sujet de son roman : Le Retour d'Ulysse (1921).

13 Asté d'Esparbès ( ?- ?) est un des fils de Georges d'Esparbès (1863-1944), dessinateur et journaliste, auteur de romans à succès et administrateur du château de Fontainebleau. Son frère, Jean d'Esparbès (1899-1968), est un peintre montmartrois ${ }^{7}$. Administrateur général de la Potinière en 1926, Asté était aussi acteur et écrivain, coscénariste des films de Duvivier, Les Roquevillard (1922) tiré de l'œuvre d'Henri Bordeaux et Les Cœurs farouches (1924). Il s'est marié le 28 décembre 1926 en l'église Saint-Louis d'Antin, avec Mlle Fernande Malpot. Au début des années 20, Asté semble s'être spécialisé dans le suivi du dadaïsme. On doit au photographe qui l'accompagne la plupart des clichés conservés sur ces manifestations. Le contraste est grand entre l'effet de notoriété que les uns et les autres apportent au groupe, et le ton souvent critique, voire franchement hostile, des articles. Ainsi le 27 mai 1920, il n'hésite pas à comparer une activité dada à une manifestation de fous. En avril 1921, c'est lui qui accompagne les Dadas dans leur visite de Paris; il figure sur la photographie de cette manifestation aux côtés de Breton, mais manifestement sans partager les comportements du groupe ${ }^{8}$. Le lendemain, il qualifie la démarche de «charentonnesque ». Le 7 juin, en décrivant «Le vernissage de l'exposition Dada » à la galerie Montaigne, son commentaire est encore plus critique. L'histoire littéraire a retenu son nom pour s'être battu avec Breton lors du banquet Saint-Paul Roux à la Closerie des Lilas le 2 juillet 1925.

Le parcours de d'Esparbès présente des points communs avec celui d'un autre collaborateur de Comœdia : Jean-Pierre Liausu. Les deux hommes collaboreront d'ailleurs à la revue mensuelle Lyrica en 1937. Liausu a été secrétaire général du Trianon-lyrique en juillet 1927. Il avait une sœur qui a été actrice sous le nom de Jeanne Lérys, qui meurt en mai 1929. Et lui aussi a un frère peintre: Camille-Paul Liausu (1894-1975). Liausu est également un ancien combattant. Dans L'image du héros, une pièce en trois actes (1930), il fait le portrait d'un jeune homme qui a conservé le culte passionné de son frère, mort au champ d'honneur. En février 1934, le général Weygand lui remet d'ailleurs la médaille militaire. Proche d'Henri Béraud, il participe à la campagne de ce dernier contre Gide 9 . S'il n'est pas préposé à la critique littéraire, il se fait connaître par une vaste enquête sur les méfaits de la cocaïne à Paris. 

drogue, son usage par les intellectuels ne représente qu'un problème marginal. Il explique que le commerce de la drogue est aux mains des Allemands qui en font une arme de revanche contre la France. La dénonciation est presque personnalisée : "Tel peintre, habitué de la rouge Rotonde, et dont le nom a une fâcheuse consonance germanique, pourrait-il nous renseigner sur ses 'soirées d'art'?» (5 novembre 1924) Il visite ensuite le «bar Tanagra», le milieu des « invertis de Montmartre", et dénonce le quartier latin comme les principaux centres de commercialisation de la cocaïne (Retaillaud-Bajac, 2005 : 151-162). Il souhaite à cette fin " épurer le quartier latin » des étrangers qui l'encombrent (22 novembre 1924). La Révolution surréaliste le dénonce comme un des «mouchards » qui se livre dans la presse à une campagne de dénigrement du surréalisme (Desnos, 1925 : 25). La suite de la carrière de l'homme est plus sordide: il deviendra éditorialiste de ParisMunicipal, journal de collaboration en 1942-1944; le 20 février 1944, il y fait un éloge d'Edouard Drumont... Il est aussi chargé de mission auprès du directeur général de l'institut d'études corporatives des spectacles, autre lieu de la collaboration (Le Matin, 15 juillet 1942). également les propos injurieux de Paul Claudel. Exaspéré par les turbulences trop sonores, très potaches de la «bande à Breton », le poète diplomate confie à un journaliste italien que le surréalisme et le dadaïsme « ont un seul sens : pédérastique » (24 juin 25). La réponse des surréalistes le 5 juillet n'est pas moins violente, mais le journal s'en fait également l'écho.

Pour autant, Comodia publie aussi des chroniques littéraires souvent plus nuancées que celles des trois journalistes que je viens de citer. Le meilleur exemple en est certainement la manière dont Louis Laloy (1874-1944) a accueilli les publications du groupe de Littérature. L'homme, il est vrai, n'était pas n'importe qui. Khâgneux à Henri IV, il a fait l'ENS d'où il est sorti avec un doctorat ès lettres. Polyglotte (il parlait français, anglais, allemand, italien, latin, russe, grec et chinois), il devint un éminent musicologue, critique musical, cofondateur du Mercure musical, enseignant à la Sorbonne puis au Conservatoire, secrétaire général de l'Opéra de Paris enfin.

Son évolution est aussi rapide que significative. Le 8 avril 1924, dans son feuilleton « Les livres ", Laloy critique Les Pas perdus de Breton qu'il a manifestement lus de manière superficielle. Il en tire le sentiment que: «M. Breton ne croit qu'au néant.» Dès novembre toutefois, il commente le Manifeste de manière plutôt positive. Il donne raison à Breton de se méfier des descriptions et du psychologisme du roman de la fin du XIX siècle. Par contre, en bon universitaire, il se méfie de l'esprit de classement trop systématique : « Le surréalisme est un système, et tout système est incomplet. » Il accorde pour finir à Breton "l'infaillibilité » de la pensée, mais insiste sur l'importance de la communiquer dans un langage accessible au lecteur, et souligne que le lien entre l'auteur et le lecteur est donné par la raison. C'est une critique humaniste traditionnelle, mais ouverte (Laloy, 1924). En janvier 1925, Laloy exprime une vraie sympathie pour les dadaïstes et les surréalistes : «Bien que profondément classique par mon goût et ma formation d'esprit, je les suis avec le plus sincère intérêt et c'est d'eux que j'attends une renaissance de la poésie français. » (Laloy, 1925) Le mois suivant, c'est du texte d'Aragon, « Une vague de rêves » qu'il rend compte non moins positivement, le 24 février 1925.

De manière plus marginale, un autre genre de réaction est également à signaler, qui emprunte les voies de l'ironie. Grâce aux caricatures qui en sont faites, et qui leur 
confèrent la légitimité d'être moqués, Aragon, Picabia ou Breton sont croqués par les dessinateurs du journal (par exemple, caricature de Breton le 24 février 1925 ou de Desnos le 7 avril). Par ailleurs, Comœdia publie aussi l'une ou l'autre parodie des pratiques de l'avant-garde telle qu'on peut la lire, par exemple sous la plume de Gabriel Timmory, dans On danse, roman parodique dont un chapitre est dédié à "l'École gagaïste " ( $1^{\mathrm{er}}$ novembre 1921). Ici encore se manifeste la réaction spontanée des professionnels du spectacle, puisque sous ce nom de plume se cache René Wahl (1870-1965), normalien et professeur de lettres, puis journaliste, romancier et auteur dramatique.

\section{Polémiques et conflits de pouvoir}

Comœedia participe également à la vie littéraire de l'avant-garde en servant de tribune aux conflits internes à ce secteur du champ littéraire. Il est en effet surprenant de constater combien ceux-ci se règlent sur la place publique, avec un maximum de publicité. Dans la période qui nous intéresse, cet usage du journal est d'abord le fait de Picabia, qui y a évidemment accès grâce à son ami Casella.

21 Le 11 janvier 1921 Marinetti lance son manifeste futuriste sur le tactilisme, qu'il lit au Théâtre de l'CEuvre. Il y annonce une nouvelle pratique artistique, un tableau composé d'un collage de matières différentes au toucher. Les dadas chahutent cette conférence. Le 18 janvier, Picabia prolonge la polémique en expliquant dans une lettre à la rédaction que le tactilisme a été inventé par Miss Clifford-Williams en 1916, lorsqu'elle a réalisé des "plâtres à toucher ", sculptures destinées à la main. C'est à New York qu'il a découvert cette innovation, et il en a fait part immédiatement à Apollinaire ; Marinetti n'est donc qu'un plagiaire. Le journal publie le même jour la réponse de Marinetti.

Comodia se présente ainsi comme une caisse de résonance des luttes de légitimité auxquelles se livre l'avant-garde. De son point de vue, la polémique fait vendre le journal et lui permet d'être au cœur de la vie artistique. Mais les artistes ont inégalement accès à ses colonnes, et Picabia dispose d'une liberté d'initiative là où Marinetti ne peut faire usage que d'un droit de réponse.

Le 11 mai 1921, Picabia annonce, dans Comodia, sa rupture avec Dada: «M. Picabia se sépare des Dadas ». Pour lui, les mouvements collectifs sont destinés à être éphémères; il faut s'en méfier dès lors qu'ils commencent à incarner des "spéculations ». Le dadaïsme était intéressant de 1913 à 1918, tant qu'il fut en évolution. Depuis qu'il a cessé de se transformer, il ne mérite plus qu'on s'y rallie. Prendre dada au sérieux, c'est enregistrer sa mort. «Dada vivra toujours! Et, grâce à lui, des marchands de tableaux feront fortune (...) » Pour sa part, Picabia pense que «L'existence n'est vraiment tolérable qu'à la condition de vivre au milieu de gens n'ayant aucune arrière-pensée, pas d'opportunistes, mais c'est demander l'impossible... ». Le 23 juin, il revient sur ce texte et précise sa pensée en une phrase :

Mes collègues m'embêtaient de plus en plus, les uns parce qu'ils se croyaient devenus des personnages importants, les autres par leur nullité, leur imbécillité ou leur muflerie. Ce sont là des choses que je ne puis supporter ; jai pris le parti de me séparer des Dadaïstes plus ou moins authentiques, afin de retrouver un peu de joie dans la vie! une mode dans laquelle l'artiste ne se retrouve pas. Mais même lorsqu'il polémique 
contre eux, Picabia ne manque pas d'adresser les mêmes reproches aux « cent-quaranteet-un dadaïstes » qui inondent le marché.

Je ne reviens pas ici sur l'aventure du "Congrès international pour la détermination des directives et la défense de l'esprit moderne " ou "Congrès de Paris » annoncé par Comoedia pour mars 1922. Celle-ci est bien connue (Sanouillet, 1972 : 319-347). Je me borne à souligner que tous les protagonistes interviennent dans le journal, de Breton à Picabia et à Tzara, sans oublier la rédaction même du quotidien. Mais il est remarquable que c'est quasiment dans les termes par lesquels Picabia se sépare des dadaïstes et dans les mêmes colonnes, que Breton tient à faire connaître sa propre désaffection. Dans " Après Dada ", il constate que les funérailles de Dada ont eu lieu vers mai 1921, soit exactement au moment où Picabia les abandonne. Il se rallie donc «à ce merveilleux détachement de toutes choses dont Picabia nous donna l'exemple ». Il achève son texte sur la certitude qu'il devra trouver un autre engagement, au risque d'être déçu encore, et en faisant sienne l'idée de Picabia d'une labilité des idées et des courants (Bonnet, $1988: 259)^{10}$. Une fois encore le journal donne la parole à la partie adverse : le 7 mars il publie la réponse de Tzara, «Les dessous de Dada » où l'écrivain roumain revendique la paternité de cette avant-garde (Tzara, 1992 : 586-588).

\section{Conclusions}

26 Il me semble que cette petite enquête conduit à quatre conclusions non dépourvues d'intérêt :

27 - Elle souligne d'abord combien les prises de positions de Breton ont été influencées par celles de Picabia. Ce constat oblige l'historien à tenir ensemble des personnalités et des domaines (peinture et littérature) que les monographies ont tendance à traiter séparément. L'histoire du surréalisme se constitue ainsi dans une dialectique permanente entre les individus, les champs artistiques, et même les zones géographiques, puisque Paris est à ce moment un point de rencontre obligé pour des créateurs venus d'Europe centrale ou passés par les États-Unis.

28 - Elle confirme aussi que la scène sur laquelle se déploient les prises de position du pôle restreint du champ littéraire outrepasse largement les limites de ce pôle ; l'avant-garde mobilise l'amplificateur médiatique de la grande presse spécialisée, et parfois même audelà. La littérature la moins destinée au grand public possède donc un versant « grand public » relativement méconnu. La capacité des auteurs de l'avant-garde à pénétrer dans les grands journaux, leur gestion de la caisse de résonance qu'ils constituent, doit être corrélée à la position qu'ils occupent dans le champ littéraire en général.

29 - On ne peut se borner à enregistrer ces prises de positions avant-gardistes dans la grande presse sans tenir compte de la logique et des positions propres à ceux qui font cette grande presse, et qui sont responsables de la mise en page, donc de la scénographie concrète de l'avant-gardisme dans le support qu'ils organisent. De ce point de vue, Comoedia hésite manifestement à se couper de l'avant-garde et parvient à maintenir un compromis plutôt efficace entre des postures incompatibles. Cette ouverture relative est facilitée par le fait que tous les journalistes ne partagent pas le même point de vue négatif sur l'avant-garde.

30 - Enfin, lorsqu'on tente d'écrire l'histoire des conflits littéraires en prenant comme angle de vue les positions de la grande presse, il apparaît que les positions habituellement 
décrites doivent être révisées. L'opposition la plus vive au surréalisme provient du milieu des anciens combattants qui se reconnaissent dans l'esthétique et les codes du théâtre privé. De leur point de vue, le dadaïsme, le surréalisme, la Nouvelle revue française, la drogue et l'homosexualité font partie du même camp antipatriotique et antitraditionnel. La structure du champ littéraire de l'époque semble ainsi s'écarter de la représentation que l'avant-garde en a donnée, pour faire entrevoir une autre réalité, sans doute plus conforme aux rapports de force réels.

\section{BIBLIOGRAPHIE}

Aron, Paul, Espagnon, Jacques (2009). Répertoire des pastiches et parodies littéraires de langue française aux XIX et $X^{\mathrm{e}}{ }^{\mathrm{e}}$ siècles. Paris : PUPS.

BANDIER, Norbert (1999). Sociologie du surréalisme (1924-1929). Paris : La Dispute.

BELLANGER, Claude (1972). Histoire générale de la presse française. Paris : PUF.

BONNET, Marguerite (1988). André Breton. Euvres complètes. Paris : Gallimard.

BOURDIEU, Pierre (1992). Les Règles de l'art. Paris : Seuil.

DESNOS, Robert (1925). « Description d'une révolte prochaine », La Révolution surréaliste, p. 25.

GRAND-CARTERET, John (1905). Zola en caricatures et en chansons. Paris : Juven.

GROJNOWSKI, Daniel. (1997). Aux commencements du rire moderne : l'esprit fumiste. Paris : Corti.

LALOY, Louis (1924) « Le surréalisme », Comœdia, 11 novembre 1924.

LALOY, Louis (1925) «A Dada », Comœedia, 20 janvier 1925.

PICABIA, Francis (1922). « Dactylocoque », Littérature, Nouvelle série, n 7, p. 3.

RETAILLAUD-BAJAC, Emmanuelle (2005). «La drogue à Paris entre les deux guerres », in Jean-Louis Robert (org). Être parisien : actes du colloque organisé par l'École Doctorale de l'Université Paris I Panthéon Sorbonne et la Fédération des Sociétés historiques et archéologiques de Paris-Île-de-France. Paris :

Publications de la Sorbonne, pp. 151-162.

SANOUILLET, Michel (1979). Dada à Paris. Paris : Flammarion.

TZARA, Tristan (1992). CEuvres complètes. Paris : Flammarion.

\section{NOTES}

1. Norbert Bandier est un des premiers à avoir souligné ce fait. Voir Bandier (1999: 86)

2. Marco Consolini, Romain Piana et Sophie Lucet (équipe EA 3959 - Institut de Recherche en Etudes Théâtrales, IRET) ont organisé deux colloques sur ce journal.

3. 5 février 1920.

4. 27 février 1920 sous la signature de J. Valmy-Baysse.

5. 29 mars 1920. 
6. Voir $1^{\mathrm{er}}$ avril, 3 avril et 4 avril 1920 et les Carnets inédits de Breton, dans CEuvres complètes.

7. Voir les renseignements biographiques que donne le site :

http://www.roussard.com/artistes/nouveaux/desparbesf/desparbesbio.html

8. 14/4/21. "Les dadas visitent Paris ", article signé Asté d'Esparbès ; voir aussi 15 avril 1921.

9. Voir : «Illusions. Le journal d'aujourd'hui », 55, 6 novembre 1924. Repris dans le BAAG, n 55, janvier 1982, pp. 414-6. C'est le 29 septembre 1924 que Béraud rejoint Comodia. Comme Gabriel Alphand s'en explique dans son éditorial du jour, cet engagement participe du repositionnement d'un journal qui cherche à conquérir plus de lecteurs, et qui attend de la publicité une part de ses recettes.

10. 2 mars 1922.

\section{RÉSUMÉS}

Cet article traite du rôle du quotidien Comoedia dans la formation du premier groupe surréaliste. Il montre que les prises de position des anciens dadaïstes se sont souvent exprimées dans la grande presse, et non pas seulement dans les petites revues du champ littéraire restreint. Sur le plan méthodologique, l'article plaide en faveur d'une analyse sociologique de l'avant-garde en relation avec les autres secteurs de la production littéraire, eux-mêmes liés aux contacts internationaux et intermédiatiques des écrivains.

This article deals with the role of the daily paper Comoedia in the formation of the first surrealist group. It shows that the stands of the former Dadaists often expressed themselves in the popular press, and not only in the small reviews of the restricted literary field. On the methodological plan, the article pleads in favor of a sociological analysis of the avant-garde in connection with the other sectors of the literary production, which were connected to the international and intermedia contacts of the writers.

\section{INDEX}

Keywords : comoedia, writers, surrealism, sociology, literature

Mots-clés : comœdia, écrivains, surréalisme, sociologie, littérature

\section{AUTEUR}

PAUL ARON

FNRS-ULB (Centre Philixte)

paron[at]ulb.ac.be 